\title{
छூ \\ Ultrashort electron bunch generation by an energy chirping cell attached rf gun
}

\author{
K. Sakaue, ${ }^{1, *}$ Y. Koshiba, ${ }^{1}$ M. Mizugaki, ${ }^{1}$ M. Washio, ${ }^{1}$ T. Takatomi, ${ }^{2}$ J. Urakawa, ${ }^{2}$ and R. Kuroda ${ }^{3}$ \\ ${ }^{1}$ Waseda University, 3-4-1, Okubo, Shinjuku, Tokyo 169-8555, Japan \\ ${ }^{2}$ KEK: High Energy Accelerator Research Organization, 1-1 Oho, Tsukuba, Ibaraki 305-0801, Japan \\ ${ }^{3}$ AIST: National Institute of Advanced Industrial Science and Technology, \\ 1-1-1, Umezono, Tsukuba, Ibaraki 305-8568, Japan
}

(Received 20 September 2013; published 19 February 2014)

\begin{abstract}
We present a new design for a rf electron gun to be used in ultrashort ( $\sim 1 \mathrm{ps})$ electron bunch generation. Using both simulation and measurement we evaluated the principle of this new type rf gun and were able to confirm an ultrashort bunch generation. During simulation, a bunch length of less than $100 \mathrm{fs}(\mathrm{rms})$ with a $100 \mathrm{pC}$ /bunch charge was confirmed at the optimum operating condition. The principle is to produce a linearly distributed longitudinal phase space by using an attached output cell specially designed for energy chirping. Such phase space distribution can be rotated by the velocity difference in the bunch. We already fabricated an energy chirping cell attached rf gun and successfully observed $0.2 \mathrm{THz}$ coherent synchrotron radiation, which corresponds to less than $500 \mathrm{fs}$ bunch. Such an electron gun can be used as a compact $\mathrm{THz}$ light source and a new electron injector with an ultrashort bunch.
\end{abstract}

DOI: 10.1103/PhysRevSTAB.17.023401

PACS numbers: 29.25.Bx, 41.60.Ap, 41.75.Ht

\section{INTRODUCTION}

We have been developing a photocathode rf electron gun in collaboration with KEK and Waseda University. At KEK-ATF (Accelerator Test Facility), our rf gun provides a high quality electron beam for the $1.3 \mathrm{GeV}$ damping ring with almost $100 \%$ injection efficiency [1]. At KEK-LUCX (Laser Undulator Compact X ray source), we have successfully produced more than 300 bunches/trains with a $1 \mathrm{nC}$ charge [2], and this is being used to study compact X-ray sources based on laser-Compton scattering [3,4]. At Waseda University, we have been performing pulse radiolysis experiments with psec bunches [5] and laser-Compton scattering experiments [6]. If an ultrashort electron bunch can be produced, the temporal resolution of the pulse radiolysis system will be further improved down to the fs range and the luminosity of laser-Compton scattering will increase. Moreover, the ultrashort electron bunch will produce other applications due to its coherent radiation. Bunches with less than 1 ps can coherently produce $\mathrm{THz}$ radiation, which can be used in application for compact radiation sources.

Our rf gun was initially designed by BNL (Brookhaven National Laboratory), named GunIV, $S$ band with 1.6-cell structure $[7,8]$. We have been improving this structure. The rf cavity shape was improved in Ref. [9] to achieve a higher $Q$ value and larger mode separation.

\footnotetext{
*kazuyuki.sakaue@aoni.waseda.jp

Published by the American Physical Society under the terms of the Creative Commons Attribution 3.0 License. Further distribution of this work must maintain attribution to the author $(s)$ and the published article's title, journal citation, and DOI.
}

Usage of a Cs-Te cathode was evaluated in Refs. [1] and [10]. Now due to the success of dark current reduction and an increase in mode separation, the 3.6-cell rf gun can successfully demonstrate $8.5 \mathrm{MeV}$ electron bunch generation [11].

All over the world ultrashort electron bunch generation is being studied using various techniques. The most successful results have been achieved at $\mathrm{x}$-ray free electron laser facilities [12,13]. At linac coherent light source, the electron source is also based on a photocathode $S$-band rf gun. There are two bunch compression systems based on the chicane technique. This needs a linearly chirped longitudinal phase space distribution to compress the bunch by using the path length difference in the chicane. They finally produce a $70 \mathrm{fs}$ electron bunch at $13.6 \mathrm{GeV}$ energy for $\mathrm{x}$-ray lasing with magnetic compressions.

In the several $\mathrm{MeV}$ to several tens of $\mathrm{MeV}$ energy region, ultrashort electron generation has been studied for the $\mathrm{THz}$ light source [14-16], pulse radiolysis experiments [17], and electron microscopes [18]. Magnetic compression, such as chicane [16] and achromatic arc section [14,15] have been widely used for the compression. An achromatic arc compression also uses an energy chirped beam to make a different path length in the section. In a photocathode rf gun, the initial bunch length can be controlled by the duration of the irradiating laser pulse. Some studies of this phenomenon have demonstrated that an ultrashort bunch could be generated initially from the cathode [18]. This method does provide an ultrashort bunch; however, the bunch charge is limited to less than $\sim 10 \mathrm{pC}$ because of the severe space charge effect near the cathode. When planning construction of a compact $\mathrm{THz}$ source, not only ultrashort but higher charge bunches are desired. 
In this paper, we present a newly designed photocathode rf gun for ultrashort bunch generation with $\sim 100 \mathrm{pC}$ charge. In Sec. II, the principle of the new rf gun and design using simulations is described. The fabrication and results of low power tests on the rf gun cavity are given in Sec. III. Experimental setup and results are described in Secs. IV and V, respectively. In Sec. VI, we summarize our results.

\section{DESIGN OF ENERGY CHIRPING CELL ATTACHED RF GUN}

\section{A. Principle of ECC-rf gun}

In order to compress the electron bunch, it is clear that a linearly modulated longitudinal phase space is essential. As we mentioned in the previous section, the magnetic compression also needs such a distribution to make a path difference in the bunch. The simplest solution for bunch compression is to use an electron gun and another separated rf cavity to modulate the energy and compress the bunch. The related scheme was discussed in [19] and [20]. Also, a gun with two separated cells was considered by [21]. Separated cells operate under different rf phases for the bunch compression. In these schemes the phase of an energy modulation can be adjusted. Such systems are naturally simpler and more flexible for generating ultrashort electron bunches with enough charge. We present, however, an energy modulation, suitable for bunch compression, which can be achieved with just the rf gun itself. This rf gun is more compact and needs less rf power for acceleration and compression. Our proposed rf electron gun achieves both acceleration and energy chirping in one accelerating cavity and is named energy chirping cell attached rf gun (ECC-rf gun).

Analysis of electrons in a rf gun has been described in $[22,23]$. Using this analysis, we present the possibilities and limitations of ECC-rf gun. We assume the rf gun structure shown in Fig. $1 . \lambda$ is the wavelength of the accelerating rf of $10.5 \mathrm{~cm}(2856 \mathrm{MHz})$. In Fig. 1, we separate the gun into two parts, part A and part B. Part A includes the cathode cell and one full cell. Part B has only one independent cell. Part A efficiently accelerates

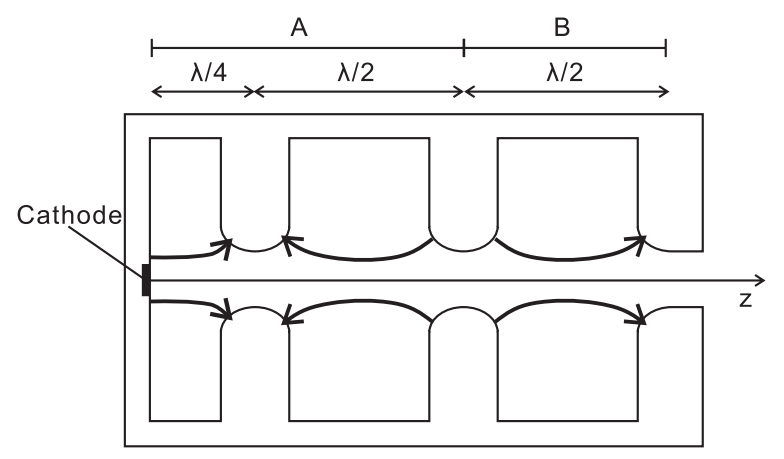

FIG. 1. Schematic of rf electron gun. electrons as in a conventional rf gun. The electric field on the $z$ axis is expressed as [22]

$$
E_{z}^{(A)}=E_{0} \cos k z \sin \left(\omega t+\phi_{0}\right) .
$$

Here $E_{0}=100 \mathrm{MV} / \mathrm{m}$ is the peak accelerating field, $k=2 \pi / \lambda, c$ is the velocity of light, $\omega=c k$, and $\phi$ is the rf phase when electrons leave the cathode. And energy of electron $\gamma$ is

$$
\gamma_{1}=1+\alpha\left\{k z \sin \phi+\frac{1}{2}[\cos \phi-\cos (\phi+2 k z)]\right\},
$$

using a dimensionless parameter of

$$
\alpha=\frac{e E_{0}}{2 m c^{2} k},
$$

and $\phi$ of

$$
\phi=\omega t-k z+\phi_{0} .
$$

In part A, the ECC-rf gun needs a small energy spread beam so we used $\phi_{0}=58^{\circ}$.

Part B plays the role of chirping the energy in the bunch. Fig. 1 shows the $\pi$-mode rf electric field, but there is no possibility of chirping the energy. Now let us assume the phase of part B can be freely chosen. If we need the maximum energy, the bunch should be accelerated on the crest of the electric field. On the other hand, if we need the maximum energy modulation, the bunch should be accelerated on the zero-crossing phase as shown in Fig. 2. The electric field of part B has to be shifted phase $\pi$ from part A for the zero-crossing phase acceleration as

$$
E_{z}^{(B)}=E_{0} \sin k z \sin \left(\omega t+\phi_{0}\right),
$$

and the resulting energy at the output of part B $\gamma_{2}$ is expressed by

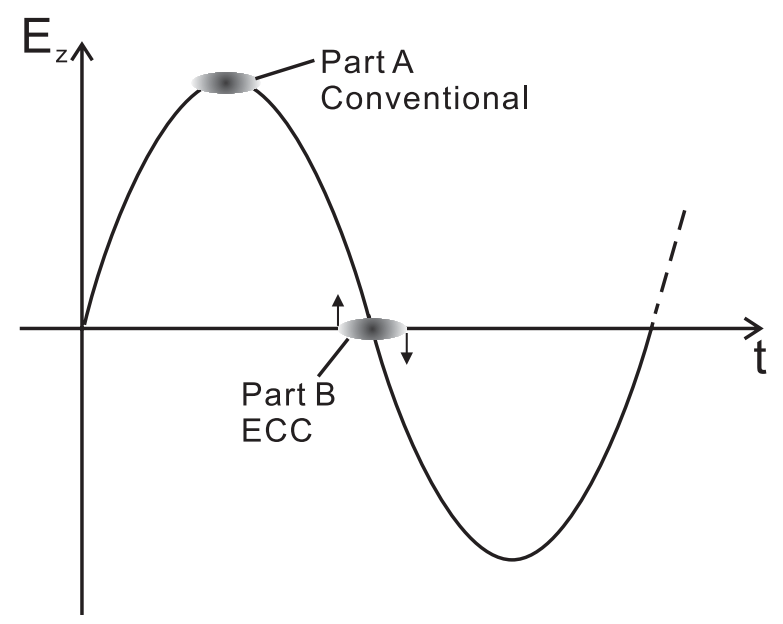

FIG. 2. Bunch acceleration by rf electric field. 


$$
\gamma_{2}=\gamma_{A}+\alpha\left\{k z \cos \phi_{e}-\frac{1}{2}\left[\sin \phi_{e}+\sin \left(\phi_{e}+2 k z\right)\right]\right\} .
$$

Here $\phi_{e}$ is the rf phase when the electron comes into part B, $\gamma_{A}$ is the energy of the electron at the end of part A, and we approximate the electron velocity by $c$ in part $\mathrm{B}$. The most linear energy chirp was achieved at phase $\phi_{e}=11^{\circ}$. We present the results of Eq. (6) as a function of position $z$ in Fig. 3 and phase space distribution in Fig. 4. In Fig. 3, electron energy $\gamma$ is plotted for $\phi_{0}=58^{\circ}, \pm 5^{\circ}$, and $\pm 10^{\circ}$. In part $\mathrm{A}$, the energies are almost the same on all five plots, and after passing through part B, the energies are separated linearly by their phase. This phenomenon can also be observed in Fig. 4. Phase space at the output of part A, shown in red, has a minimum energy spread. At the output of part $\mathrm{B}$, the phase space is modulated by the energy chirping cell, shown in blue, into a linear distribution, then the rotation of this phase space by the velocity difference between each electron causes the bunch compression as

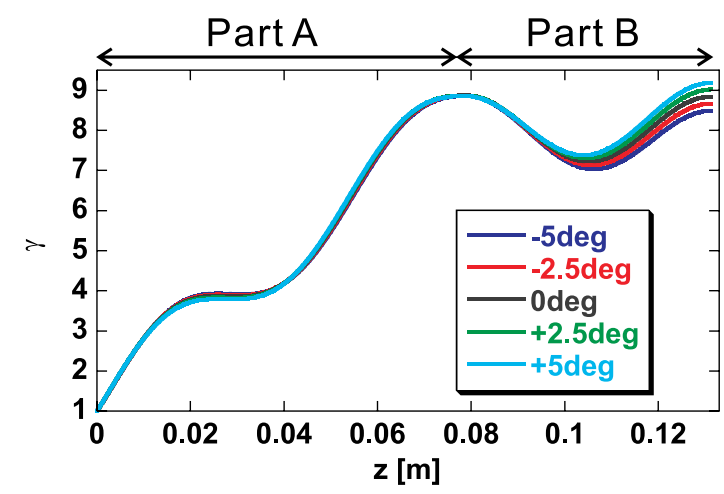

FIG. 3 (color online). Evolution of $\gamma$ in the rf gun for the cases of $\phi_{0}, \pm 2.5^{\circ}$, and $\pm 5^{\circ}$.

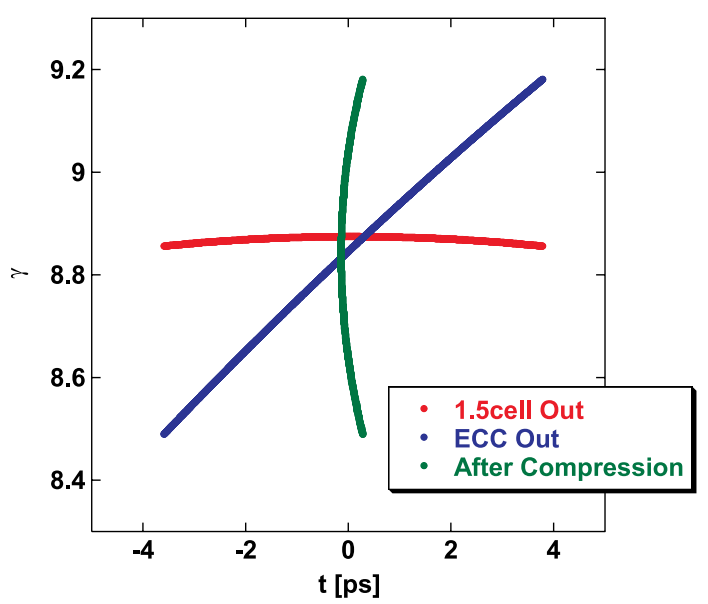

FIG. 4 (color online). Phase space distribution at the end of part A (red), end of part B (blue), and at $2.33 \mathrm{~m}$ away from the cathode. shown in green at $2.33 \mathrm{~m}$ away from the cathode. Here, we assumed a 4 ps (rms) gaussian distribution for the initial electron bunch. As a result, a $39 \mathrm{fs}$ (rms) bunch can be achieved at $z=2.33 \mathrm{~m}$ in this calculation. Though this analytical calculation does not include space charge effects and electric field distribution on the $x$ and $y$ axis, it gives the limitation of this compression method by the timedependent electric field acceleration and modulation.

\section{B. Design of ECC-rf gun by Superfish and general particle tracer}

First we present the latest rf gun structure (Superfish image) in Fig. 5 [9] and our newly designed ECC-rf gun structure in Fig. 6. It is obvious that the ECC-rf gun cavity has one more cell (ECC) after the 1.6-cell rf gun. Figure 6 is the practical solution to realize the discussion in the previous paragraph. To optimize the design of the ECC-rf gun, we iterated by changing the structure of the ECC on Superfish [24] and performing a beam tracing

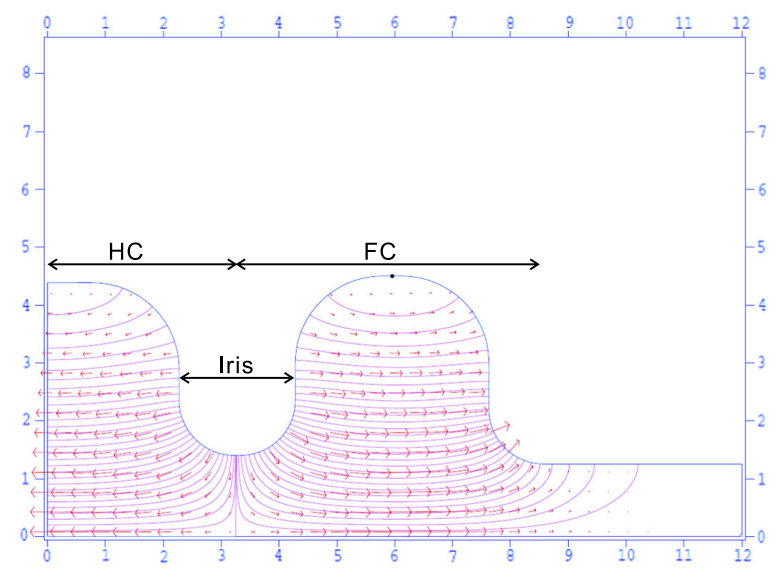

FIG. 5 (color online). Profile of a conventional 1.6-cell rf gun. Designed to obtain a minimum energy spread.

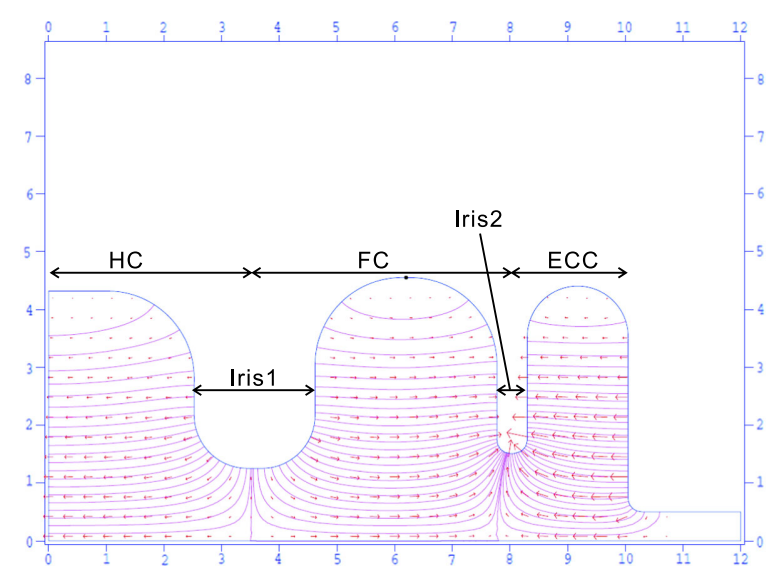

FIG. 6 (color online). Profile of an ECC-rf gun. ECC is added to a conventional rf gun shape. The parts, half cell (HC), full cell (FC), ECC, iris 1, and iris 2, are described. 
TABLE I. Design parameters of ECC-rf gun cavity.

\begin{tabular}{lcccc}
\hline \hline Parameter & $Q$ value & $\mathrm{E}_{h}$ & $\mathrm{E}_{f}$ & $E_{e}$ \\
\hline Value & 13290 & 1 & 1 & 1.5 \\
\hline \hline
\end{tabular}

simulation by a general particle tracer (GPT) [25]. We surveyed the iris 2 width, ECC length and output port aperture while maintaining the resonant frequency, in order to achieve the shortest bunch length in the GPT. The ECC-rf gun is considered to operate at $\pi$ mode. Shortening of the iris 2 and the ECC length are both effective in chirping the energy. It is clear that such a structure decreases the $Q$ value. Thus, an ECC-rf gun has a small output port, which plays an important role in increasing the $Q$ value. Such constraints made ECC as a half cell of the rf field.

The parameters of an ECC-rf gun cavity after optimization are shown in Table I. As shown in Table I, the designed cavity has a sufficient $Q$ value. In order to achieve enough energy chirping in ECC, the field distribution is $1: 1: 1.5$. This is also an appropriate value according to the GPT simulation. The GPT simulation beam line consists of an ECC-rf gun electric field produced by the Superfish, a solenoid magnet for emittance compensation and drift spaces. The results of the GPT simulations are shown in Figs. 7 and 8, and parameters for the calculation are shown in Table II. Though we mentioned $58^{\circ}$ was the minimum energy spread phase in the previous paragraph the most compressed rf phase was actually $20^{\circ}$. This is due to the half cell length difference. The optimized half cell length was 0.6 cell, while the numerical calculation uses 0.5 cell for simplify. The phase space distribution at the ECC-rf gun output (top) and at $2.3 \mathrm{~m}$ away from the cathode (bottom) are shown in Fig. 7. The linear distributed phase space was realized at the gun output. Such a phase space distribution brings about bunch compression by velocity bunching in the free space of $2.3 \mathrm{~m}$. The bunch length transition as a function of distance is shown in Fig. 8. The bunch length is compressed as it drifts down to $88.4 \mathrm{fs}$ (rms) at $2.3 \mathrm{~m}$ from the cathode. The $2.3 \mathrm{~m}$ measurement is almost the same distance as the model calculation in the previous paragraph; thus the designed ECC-rf gun almost realizes the simulated configuration. It is important to note that a bunch with sufficient $100 \mathrm{pC} /$ bunch charge would be compressed down to less than $100 \mathrm{fs}$ (rms), only with a single gun cavity.

\section{Results and discussions of beam tracing simulations}

We summarize the results of beam tracing simulations in this paragraph. The results of the simulation are listed in Table III. A PARMELA simulation [24] was also performed as a cross-check. As shown in Table III, the results from each simulation are almost the same. It means that these results are more probable than those from one simulation result. Another point to note is the emittance
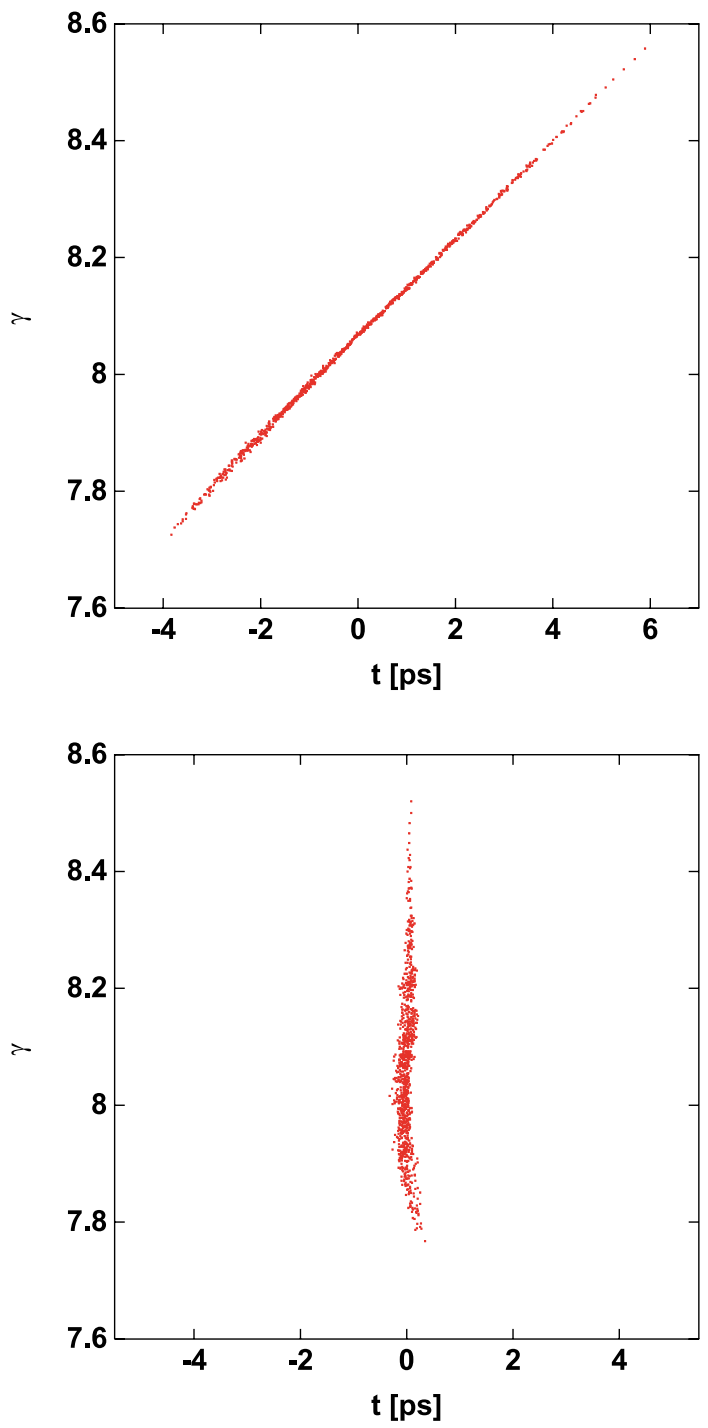

FIG. 7 (color online). Phase space distribution at the output of ECC-rf gun (top) and most compressed point (bottom) calculated by GPT.

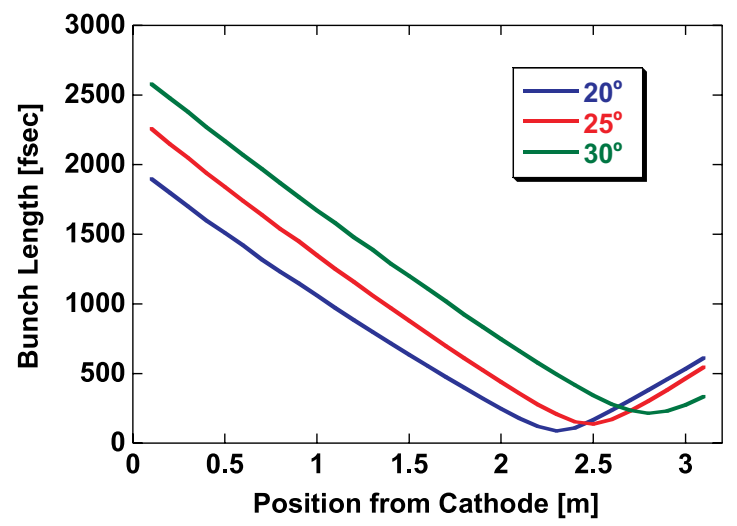

FIG. 8 (color online). Evolution of the bunch length by GPT. Three of laser injection rf phase are shown. The optimum phase was $20^{\circ}$, and a $88.4 \mathrm{fs}$ (rms) ultrashort bunch was achieved at $z=2.3 \mathrm{~m}$. 
TABLE II. Simulation parameters.

\begin{tabular}{ll}
\hline \hline Parameter & \multicolumn{1}{c}{ Value } \\
\hline Charge & $100 \mathrm{pC} /$ bunch \\
$\mathrm{E}_{z}$ at cathode & $100 \mathrm{MV} / \mathrm{m}$ \\
Injection phase & $20^{\circ}$ \\
Initial bunch length & $4.3 \mathrm{ps}(\mathrm{rms})$ \\
Initial beam size & $400 \mu \mathrm{m}(\mathrm{rms})$ \\
Solenoid field & $1050 \mathrm{G}$ \\
\hline \hline
\end{tabular}

TABLE III. Results of ECC-rf gun simulation by GPT and PARMELA.

\begin{tabular}{lcc}
\hline \hline & GPT & PARMELA \\
\hline Energy & $4.24 \mathrm{MeV}$ & $4.34 \mathrm{MeV}$ \\
Minmum bunch length & $88.4 \mathrm{fs}(\mathrm{rms})$ & $85.9 \mathrm{fs}(\mathrm{rms})$ \\
Most compressed distance & $2.30 \mathrm{~m}$ & $2.36 \mathrm{~m}$ \\
Normalized emittance & $4.39 \pi \mathrm{mm} \mathrm{mrad}$ & $4.27 \pi \mathrm{mm} \mathrm{mrad}$ \\
\hline \hline
\end{tabular}

( $r$ direction) is less than $5 \pi \mathrm{mmmrad}$ with lower than 100 fs bunch length. Therefore, the ECC-rf gun can be regarded as not only a very high quality electron gun but an ultrashort photoinjector for a linac system with ultrashort bunch and small energy spread.

\section{CAVITY FABRICATION AND LOW LEVEL RF CHARACTERISTICS}

\section{A. Cavity fabrication}

To fabricate an ECC-rf gun cavity as shown in Fig. 6, we separated it into four parts in order to machine it by diamond turning. The parts were fabricated from Hitachi oxygen-free high thermal conductivity copper with hot isostatic pressing. Four parts were fabricated using diamond turning to achieve the desired frequency and field balances. After frequency tuning, the four parts were brazed with gold and copper into one cavity. From the boundary conditions of our accelerator, we slightly changed the parameters of the ECC-rf gun cavity for practical tests. The fabricated cavity has sufficient characterizations. Figure 9 shows a photograph of the fabricated ECC-rf gun cavity. The end plate on the half cell has a port for a Cs-Te evaporated cathode plug insertion. The high power rf from klystron is fed through the waveguide brazed on the upper side. The waveguide is located on the full cell. The coupling between the cavity and the waveguide was adjusted using the pin placed on the waveguide. At the final stage of fabrication, the pin was brazed after adjusting the coupling.

\section{B. Low level rf characteristics of the ECC-rf gun}

After the fabrication, we performed a low level rf measurement using a network analyzer (Agilent N5230 A). The results of measurement and comparison with Superfish simulation are shown in Table IV. The

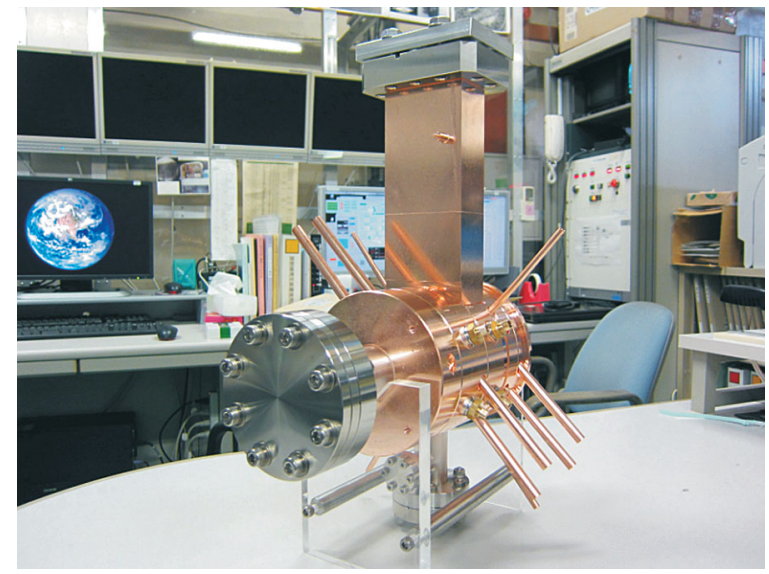

FIG. 9 (color online). Photograph of ECC-rf gun cavity. Cathode insertion port (near side), beam port (far side), rf waveguide (top), and vacuum pumping port (bottom). Water cooling pipes and rf tuners can be seen around the cavity.

design value described in the previous section was optimized for $100 \mathrm{MV} / \mathrm{m}$ electric field at a cathode surface. However, we have a boundary condition on klystron output power of $10 \mathrm{MW}$ at Waseda University, so that we cannot achieve $100 \mathrm{MV} / \mathrm{m}$. Therefore we made a minor change to the ECC-rf gun parameters in order to observe the synchrotron radiation (SR) in the $\mathrm{THz}$ range. In other words, we modified the ECC-rf gun parameters to obtain sufficient accelerating voltage for $10 \mathrm{MW}$ rf power. The expected beam parameters are shown below. The results listed in Table IV show good agreement with Superfish calculation. For the $Q$ value, the coupling hole was made smaller but the measured value of more than 10000 was enough. For the coupling coefficient $\beta$, almost perfect matching was made due to the fine-tuning of the adjusting pin.

TABLE IV. Results of low power rf characterization compared to Superfish.

\begin{tabular}{lcc}
\hline \hline & Measured & Superfish \\
\hline$Q$ value & 10860 & 13930 \\
Coupling $\beta$ & 0.92 & $\cdots$ \\
$\mathrm{E}_{h}$ & 0.9 & 1 \\
$\mathrm{E}_{f}$ & 1 & 1 \\
$\mathrm{E}_{e}$ & 1.23 & 1.22 \\
\hline \hline
\end{tabular}

TABLE V. Expected electron beam from the fabricated ECC-rf gun at Waseda accelerator system.

\begin{tabular}{lc}
\hline \hline Energy & $3.7 \mathrm{MeV}$ \\
\hline Minimum bunch length & $180 \mathrm{fs}(\mathrm{rms})$ \\
Most compressed distance & $3.02 \mathrm{~m}$ \\
Normalized emittance & $5.7 \pi \mathrm{mm} \mathrm{mrad}$ \\
\hline \hline
\end{tabular}




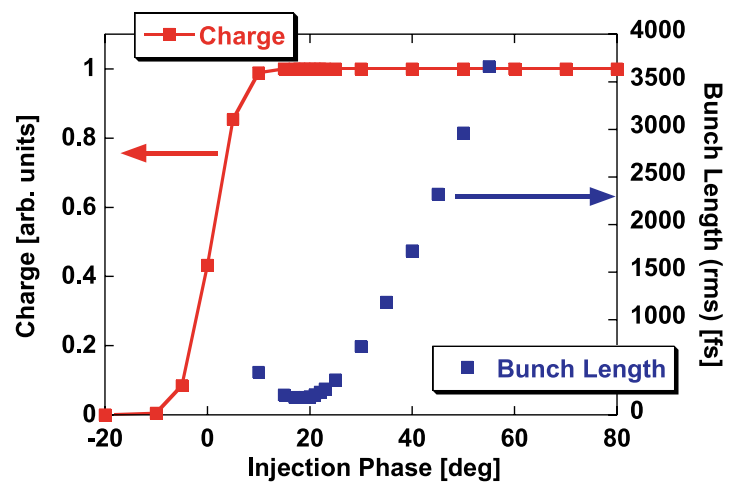

FIG. 10 (color online). Expected bunch length as a function of injection phase at $z=3.0 \mathrm{~m}$. The shortest bunch was $180 \mathrm{fs}$ (rms) at the phase of $19^{\circ}$.

\section{Simulation results compared to a fabricated ECC-rf gun}

In Sec. II, the optimized ECC-rf gun was described, but the gun which we used was slightly modified as mentioned above. Therefore the expected beam parameters from our accelerator system were recalculated. Table $\mathrm{V}$ shows the expected beam parameters. $180 \mathrm{fs}(\mathrm{rms})$ bunch length with 3.7 MeV energy would be achieved under our accelerator conditions. It is short enough to generate coherent SR in the $\mathrm{THz}$ range. The distance from cathode increased from 2.3 to $3.0 \mathrm{~m}$ because the ECC field strength was decreased, i.e. the energy modulation strength was decreased by the modification from the optimum.

Figure 10 shows the rms bunch length at $3.0 \mathrm{~m}$ position as a function of the laser injection phase. In the experiment, the optimum injection phase was sought. Around $20^{\circ}$ of $\mathrm{rf}$ phase, a lower than 200 fs ultrashort bunch should be produced by the ECC-rf gun.

\section{EXPERIMENTAL SETUP}

\section{A. Accelerator system at Waseda University}

We have an accelerator system for rf gun development [10] and for studying its applications [5,6]. The accelerating structure is only the rf gun cavity. From a $10 \mathrm{MW}$ rf pulse fed from the klystron, the conventional 1.6-cell rf gun can produce a short bunch electron beam with up to $6 \mathrm{MeV}$. Our accelerator system is based on an $S$-band $2856 \mathrm{MHz}$ frequency. The rf pulse repetition is up to $25 \mathrm{pps}$ with up to $4 \mu$ s width.

Concerning the pulsed laser for a photocathode, a Nd: YLF mode-locked oscillator and amplifier is used. A $119 \mathrm{MHz}$ mode-locked oscillator provides a ps pulse with $8.4 \mathrm{~ns}$ pulse spacing, then the pulse picker picks the desired number of pulses of $1 \sim 100$ pulses/train. After the pulse picker, a two-stage amplifier system is used. The $\mathrm{Yb}$-doped fiber-based amplifier has more than 100 times gain as the preamplifier, and the rod type Nd:YLF main amplifier has a gain of more than 10000 times. After the amplification,

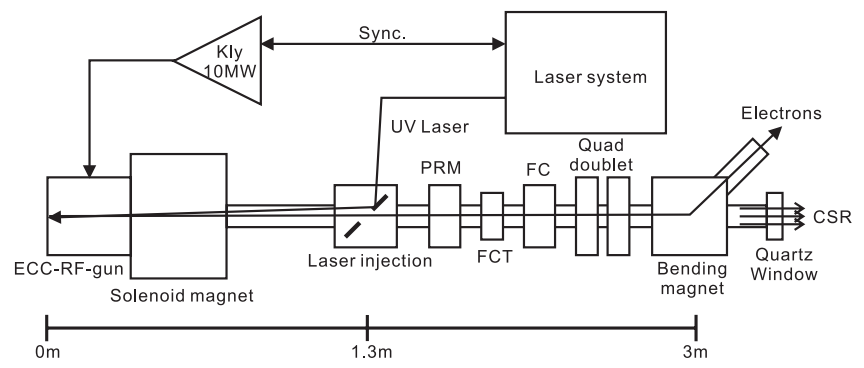

FIG. 11. Experimental setup for the ECC-rf gun.

LBO (SHG) and BBO (FHG) crystals convert the wavelength of the laser from $1047 \mathrm{~nm}$ to $262 \mathrm{~nm}$. The pulse width is $10 \mathrm{ps}$ (FWHM) measured by the autocorrelation method. The pulse energy is $1 \mu \mathrm{J} /$ pulse but the photocathode has quantum efficiency of about $0.1 \%$, which produces an electron bunch with more than $1 \mathrm{nC}$ charge. The $119 \mathrm{MHz}$ mode-locked oscillator is synchronized to the $\mathrm{rf}$ frequency of $2856 \mathrm{MHz}$ with an accuracy of $0.3 \mathrm{ps}$ (rms). In this experiment, we just used a single bunch operation to simplify the measurement.

\section{B. Beam line setup}

For the ECC-rf gun studies, we built a beam line as shown in Fig. 11. A measurement of $10 \mathrm{MW}$ rf power produces a $75 \mathrm{MV} / \mathrm{m}$ electric field on the cathode surface. A pulsed UV laser irradiates perpendicularly to the photocathode from the middle of the beam line with a centerholed aluminum mirror. The ECC-rf gun is mounted on a solenoid magnet for emittance compensation. A profile monitor screen (PRM) for profile measurement, a fast current transformer (FCT), and a faraday cup for charge measurement are located on the beam line. Beam energy is measured by a bending magnet and PRM. A bending magnet is located at around $3 \mathrm{~m}$ from the cathode, which is the point at which the electron bunch has the shortest length in the simulation. The electron bunch generates the SR by being bent. We performed a SR intensity measurement in the THz region, through a $z$-cut quartz window with wedge angle of $0.67^{\circ}$, which has sufficient transmittance. In this paper, we performed a simple spectroscopy of a coherent SR (CSR) using the narrow band Schottky barrier diode (SBD) for bunch length estimation.

\section{EXPERIMENTAL RESULTS AND DISCUSSIONS}

\section{A. Charge and energy measurement}

We first performed charge and energy measurements as a function of the laser injection rf phase. The ECC-rf gun gives the bunch a linear energy chirp. Therefore, beam energy as a function of the $\mathrm{rf}$ phase provides very important information. Figure 12 shows the results of the energy measurement. The graph is plotted as a function of the laser injection rf phase. The measured energies (dots) show good agreement with the simulation results (solid line). In the 


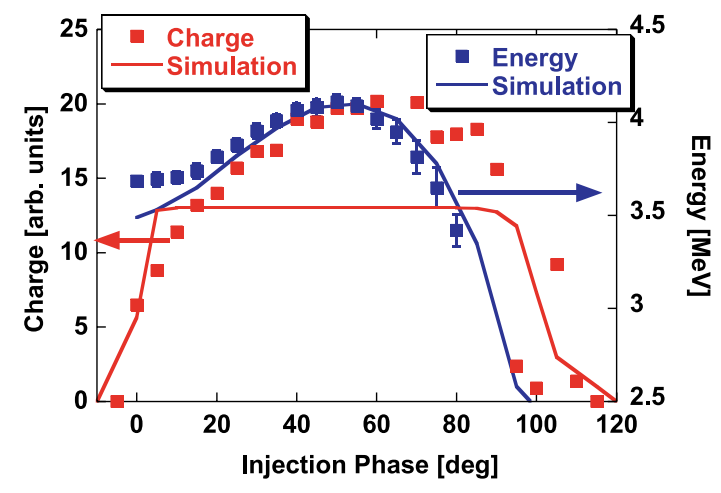

FIG. 12 (color online). Results of charge and energy measurement as a function of injection phase. The dots and lines show measurement results and simulation results, respectively. Measurement and simulation show good agreement.

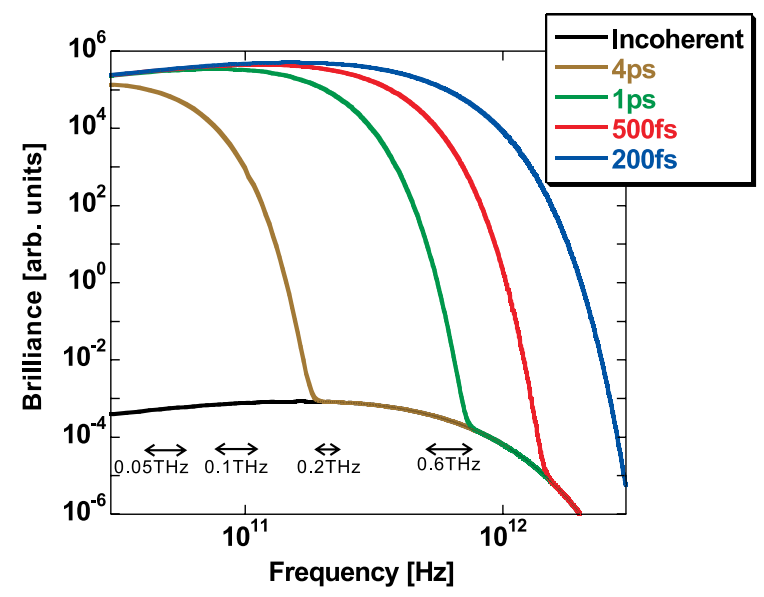

FIG. 13 (color online). Calculated synchrotron radiation spectrum by a $3.7 \mathrm{MeV}$ electron bent by a $0.14 \mathrm{~T}$ magnetic field. Coherent enhancement of various bunch lengths are plotted on the incoherent spectrum.

simulation, the charge profile was a top-hat shape because the charge calculation does not include the Schottky effect from the rf electric field. It is clear that the ECC-rf gun works correctly as in the design simulation. We consider that this plot provides a proof of the ECC-rf gun concept.

\section{B. Coherent synchrotron radiation observation}

We estimated the resulting bunch length by measuring the CSR. The expected CSR spectrum of each bunch length is shown in Fig. 13. A $3.7 \mathrm{MeV}$ energy electron can generate $\mathrm{THz}(0.1 \sim 10 \mathrm{THz})$ radiation. As shown in Fig. 13, the cutoff frequency of SR in our setup, with a bending magnet strength of $0.14 \mathrm{~T}$, was about $0.6 \mathrm{THz}$. In addition, SR was observed in a straight line in our current setup, which indicates a lower cutoff frequency $(\sim 0.3 \mathrm{THz})$ due to the fringe field of the bending magnet. In the spectrum, coherent enhancement is observed for each bunch length. If we assume the bunch shape to be a Gaussian distribution, the critical frequency $\omega_{c}$ is expressed as

$$
\omega_{c}=\frac{\pi c}{\sigma_{z}},
$$

and the bunch form factor is

$$
f(\omega)=e^{-\frac{\omega^{2} \sigma_{z}^{2}}{2 c^{2}}}
$$

where $\sigma_{z}[\mathrm{~m}]$ is the bunch length. Using this equation, we can obtain the Fig. 13 spectrum. Shorter bunches can produce CSR at higher frequencies. In this paper, we estimate the bunch length by comparing the measurement results of various narrow band SBDs and Fig. 13.

SBDs' specifications, which we used in this experiment, are listed in Table VI. We used three kinds of SBD and another is BPF (band pass filter) with SBD. They are named by the center frequency as $0.05 \mathrm{THz}, 0.1 \mathrm{THz}$, $0.2 \mathrm{THz}$, and $0.6 \mathrm{THz}$. The $0.2 \mathrm{THz}$ detector consists of $0.1 \mathrm{THz}$ SBD and $0.2 \mathrm{THz}$ BPF. The $0.1 \mathrm{THz}$ SBD has low sensitivity at $0.2 \mathrm{THz}$. From the sensitivity of these SBDs, it can be regarded that SBDs are unable to observe the radiation signals without coherent enhancement.

The waveforms from the $0.1 \mathrm{THz}$ SBD and FCT electron charge signal are shown in Fig. 14. A single pulse from the $0.1 \mathrm{THz}$ SR was observed from about a $100 \mathrm{pC}$ electron bunch. This waveform intensity of $67.6 \mathrm{mV}$ corresponds to $0.13 \mathrm{~mW}$ by SBD sensitivity. We shall discuss the bunch length later but for now we assume the bunch length to be $500 \mathrm{fs}$, and the peak power of $\mathrm{THz}$ radiation observed to be $0.54 \mathrm{~W}$ within the SBD sensitivity range of $0.075-$ $0.11 \mathrm{THz}$. Extrapolating to a total bandwidth of the $\mathrm{THz}$ range, $\mathrm{kW}$ class high peak power radiation could be generated.

In order to confirm the CSR generation, THz intensity is plotted as a function of bunch charge in Fig. 15. The dotted

TABLE VI. Specification of Schottky barrier diodes.

\begin{tabular}{lcccc}
\hline \hline Name & $0.05 \mathrm{THz}$ & $0.1 \mathrm{THz}$ & $0.2 \mathrm{THz}$ & $0.6 \mathrm{THz}$ \\
\hline Model & DXP-19 & FAS-10SF-01 & BPF $^{\mathrm{a}}$ & WR1.5ZBD \\
Mfr & Millitech & Wisewave & TYDEX & VDI \\
Sensitive range & $0.04-0.06 \mathrm{THz}$ & $0.075-0.11 \mathrm{THz}$ & $0.18-0.22 \mathrm{THz}$ & $0.5-0.75 \mathrm{THz}$ \\
Sensitivity $\mathrm{mV} / \mathrm{mW}$ & 1000 & 500 & $\cdots$ & 750 \\
\hline \hline
\end{tabular}

\footnotetext{
${ }^{\mathrm{a}}$ Use $0.2 \pm 0.02 \mathrm{THz}$ band pass filter with FAS-10-SF-01.
} 


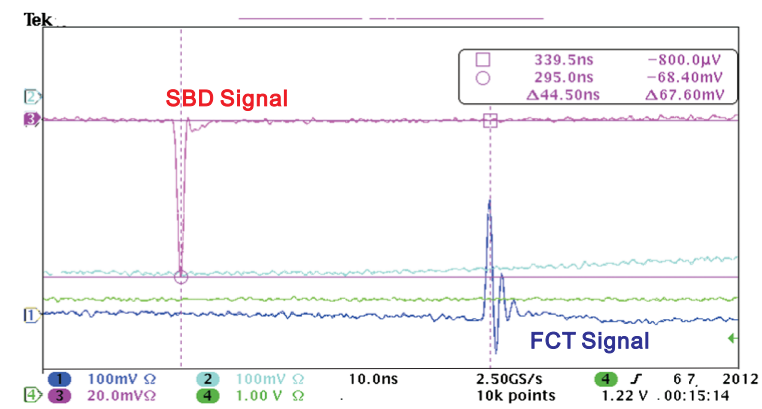

FIG. 14 (color online). Waveform of SR detected by $0.1 \mathrm{THz}$ SBD (pink) and bunch charge by FCT (blue). The pulse height of SBD waveform was $67.6 \mathrm{mV}$ from about $100 \mathrm{pC} /$ bunch electrons.

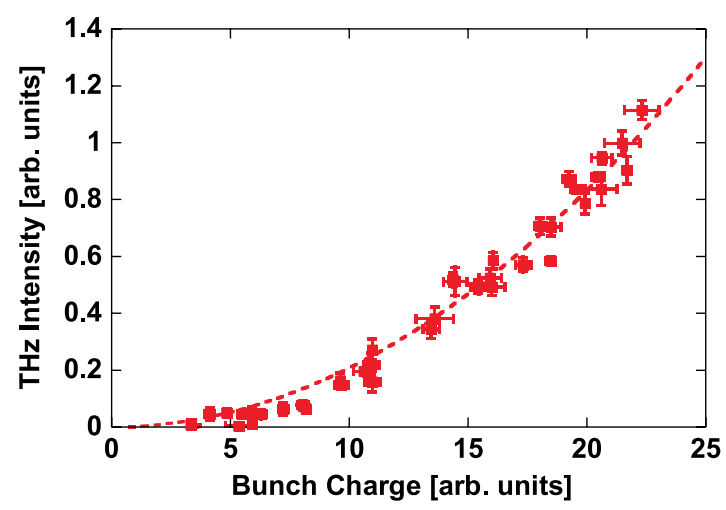

FIG. 15 (color online). $\quad 0.2 \mathrm{THz}$ intensity as a function of bunch charge. The dotted line is the best fit for the experimental data. SR intensity was proportional to the square of bunch charge, i.e. coherent enhancement was observed.

line is the best fit for the plots. The $0.2 \mathrm{THz}$ radiation intensity increases with the square of the bunch charge, i.e. $0.2 \mathrm{THz}$ SR from the bunch is coherently enhanced.

\section{Bunch length estimation by CSR}

It is very important to adjust the laser injection rf phase for an ECC-rf gun. As shown in Fig. 10, only around $20^{\circ}$ can produce lower than $300 \mathrm{fs}$ bunch. CSR intensities as a function of laser injection rf phase are shown in Fig. 16. We tried all SBDs and observed a radiation signal except at $0.6 \mathrm{THz}$. We considered that there are two reasons for this failure. One is the small aperture of $0.6 \mathrm{THz} \mathrm{SBD}$ of $0.381 \times 0.191 \mathrm{~mm}$. The other is the fringe field of the bending magnet as we mentioned above. Considering the intensity profile of CSR, all the peak phases were $20^{\circ}$. Also, the intensity profile gets narrower as the radiation frequency gets higher. This certainly agrees with the simulation result of Fig. 10.

By following the successful results of $0.2 \mathrm{THz}$ CSR observation (Fig. 15), agreement with simulation on beam energy (Fig. 12), and radiation intensity profile (Fig. 16),

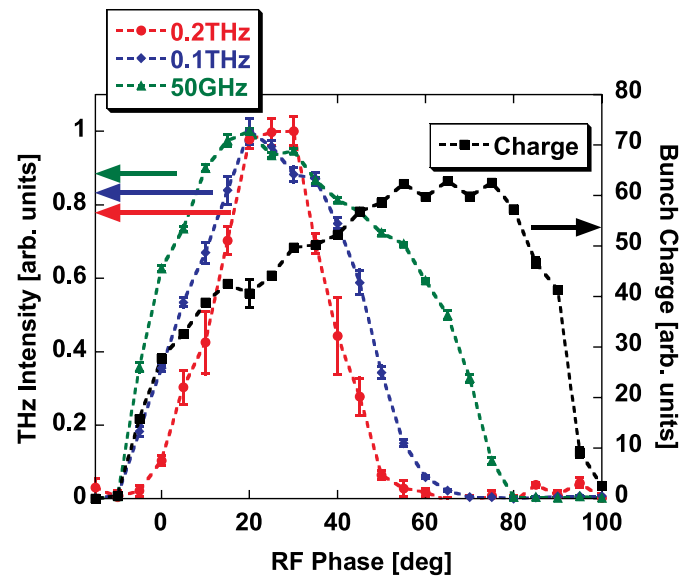

FIG. 16 (color online). CSR intensity as a function of the injection phase detected by $0.05 \mathrm{THz} \mathrm{SBD}$ (green), 0.1 THz SBD (blue), and $0.2 \mathrm{THz} \mathrm{BPF}+\mathrm{SBD}$ (red). Bunch charge is also plotted as a reference. The peak of $\mathrm{THz}$ intensity was around $20^{\circ}$ and the profile became narrower as the radiation frequency grew higher.

we confirmed that an ECC-rf gun compresses the bunch. As for bunch length, we cannot give an absolute value from these measurements. However, considering the CSR spectrum (Fig. 13), $0.2 \mathrm{THz}$ CSR observation corresponds to a bunch length of $500 \mathrm{fs}$ (rms). Moreover, the $0.2 \mathrm{THz}$ intensity profile as a function of the injection phase has about $\pm 10^{\circ}$ width and it indicates that higher frequency radiation is coherently produced at the optimum phase of $20^{\circ}$. We therefore believe that the bunch was correctly compressed down to less than $200 \mathrm{fs}$ at the optimum operating conditions.

\section{CONCLUSION}

In this paper, we presented a newly designed rf electron gun named ECC-rf gun. The principle of this ECC-rf gun is to attach an additional cell to chirp the bunch energy and achieve a linear phase space distribution. At the several $\mathrm{MeV}$ range, the velocity difference compresses the bunch. The ultimate limitation on bunch length comes from the linearity of the rf field, which is $39 \mathrm{fs}$ (rms) for a $4 \mathrm{ps}$ (rms) initial bunch. The ECC-rf gun design was performed by using simulation codes. A lower than 100 fs (rms) ultrashort bunch was achieved under the optimum parameters.

In accordance with the successful design, we fabricated the ECC-rf gun cavity. The ECC-rf gun tests were carried out at Waseda University. In our accelerator system, the optimum operating condition could not be achieved but a $180 \mathrm{fs}$ bunch length was expected from the simulation. As an experimental result, $0.54 \mathrm{~W}$ peak power coherent synchrotron radiation was observed at a $100 \mathrm{pC}$ bunch charge in the range of only around $0.1 \mathrm{THz}(0.075-$ $0.11 \mathrm{THz}$ ). The SR spectrum measurement was performed by using various Schottky barrier diode detectors. A 
measurement of $0.2 \mathrm{THz}$ with $20 \%$ bandwidth CSR was successfully observed, which corresponds to a bunch length of less than $500 \mathrm{fs}$ rms. We believe that the bunch from the ECC-rf gun was correctly compressed down to less than $200 \mathrm{fs}$ (rms), but further bunch length diagnostics are required for confirmation.

In the near future, we are planning to directly observe the bunch length by using a rf deflector [26]. Direct measurement will provide not only an absolute value for bunch length but also the longitudinal profile of the ECC-rf gun bunch. $\mathrm{THz}$ radiation may be used in many applications such as $\mathrm{kW}$ class $\mathrm{THz}$ pulses for the $\mathrm{THz}$ time domain spectroscopy and/or nonlinear dynamics studies. We are considering constructing a compact high power $\mathrm{THz}$ source using an ECC-rf gun.

\section{ACKNOWLEDGMENTS}

The authors would like to thank Mr. Kudo of the KEK machining center for providing his valuable support on $\mathrm{rf}$ gun fabrication. This work was supported by JSPS Grantin-Aid for Young Scientists (B) 23740203 and Scientific Research (A) 10001690.

[1] N. Terunuma et al., Nucl. Instrum. Methods Phys. Res., Sect. A 613, 1 (2010).

[2] A. Deshpande, S. Araki, M. Fukuda, K. Sakaue, N. Terunuma, J. Urakawa, and M. Washio, Phys. Rev. ST Accel. Beams 14, 063501 (2011).

[3] K. Sakaue, M. Washio, S. Araki, M. Fukuda, Y. Higashi, Y. Honda, T. Omori, T. Taniguchi, N. Terunuma, J. Urakawa, and N. Sasao, Rev. Sci. Instrum. 80, 123304 (2009).

[4] K. Sakaue, T. Aoki, M. Washio, S. Araki, M. Fukuda, N. Terunuma, and J. Urakawa, AIP Conf. Proc. 1466, 272 (2012).

[5] Y. Hosaka, R. Betto, K. Sakaue, R. Kuroda, S. Kashiwagi, K. Ushida, and M. Washio, Radiat. Phys. Chem. 84, 10 (2013).

[6] K. Sakaue, T. Gowa, H. Hayano, Y. Kamiya, S. Kashiwagi, R. Kuroda, A. Masuda, R. Moriyama, J. Urakawa, K. Ushida, X-J. Wang, and M. Washio, Radiat. Phys. Chem. 77, 1136 (2008).

[7] X. J. Wang and I. Ben-Zvi, in Proceedings of the Particle Accelerator Conference Vancouver, BC, Canada, 1997 (IEEE, New York, 1997), pp. 2793-2795.

[8] D. T. Palmer, X. J. Wang, R. H. Miller, M. Babzien, I. Ben-Zvi, C. Pellegrini, J. Sheehan, J. Skaritka, H. Winick, M. Woodle, and V. Yakimenko, in Proceedings of the
Particle Accelerator Conference Vancouver, BC, Canada, 1997 (IEEE, New York, 1997), pp. 2687-2689.

[9] A. Deshpande, S. Araki, M. Fukuda, K. Sakaue, N. Terunuma, J. Urakawa, N. Sasao, and M. Washio, Nucl. Instrum. Methods Phys. Res., Sect. A 600, 361 (2009).

[10] K. Sakaue, H. Hayano, S. Kashiwagi, R. Kuroda, A. Masuda, T. Suzuki, T. Takatomi, N. Terunuma, J. Urakawa, and M. Washio, Nucl. Instrum. Methods Phys. Res., Sect. B 269, 2928 (2011).

[11] T. Aoki, K. Sakaue, M. Washio, A. Deshpande, J. Urakawa, M. Fukuda, N. Kudo, N. Terunuma, and T. Takatomi, in Proceedings of the 2nd International Particle Accelerator Conference, IPAC-2011, San Sebastián, Spain, 2011 (EPS-AG, Spain, 2011), pp. 110-112.

[12] P. Emma et al., Nat. Photonics 4, 641 (2010).

[13] T. Ishikawa et al., Nat. Photonics 6, 540 (2012).

[14] R. Kuroda, N. Sei, M. Yasumoto, H. Toyokawa, H. Ogawa, M. Koike, and K. Yamada, Infrared Phys. Technol. 51, 390 (2008).

[15] K. Kan, J. Yang, A. Ogata, T. Kondoh, K. Norizawa, and Y. Yoshida, Appl. Phys. Lett. 99, 231503 (2011).

[16] Y. Shen, T. Watanabe, D. A. Arena, C.-C. Kao, J. B. Murphy, T. Y. Tsang, X. J. Wang, and G. L. Carr, Phys. Rev. Lett. 99, 043901 (2007).

[17] J. Yang, T. Kondoh, K. Kan, and Y. Yoshida, Nucl. Instrum. Methods Phys. Res., Sect. A 629, 6 (2011).

[18] Y. Murooka, N. Naruse, S. Sakakihara, M. Ishimaru, J. Yang, and K. Tanimura, Appl. Phys. Lett. 98, 251903 (2011).

[19] A. Doria, G. P. Gallerano, E. Giovenale, S. Letardi, G. Messina, and C. Ronsivalle, Phys. Rev. Lett. 80, 2841 (1998).

[20] S. G. Biedron, J. W. Lewellen, S. V. Milton, N. Gopalsami, J. F. Schneider, L. Skubal, Y. Li, M. Virgo, G. P. Gallerano, A. Doria, E. Giovenale, G. Messina, and I. P. Spassovsky, Proc. IEEE 95, 1666 (2007).

[21] H. Hama, H. Tanaka, N. Kumagai, M. Kawai, F. Hinode, T. Muto, K. Nanbu, T. Tanaka, K. Kasamsook, K. Akiyama, and M. Yasuda, New J. Phys. 8, 292 (2006).

[22] K-J. Kim, Nucl. Instrum. Methods Phys. Res., Sect. A 275 , 201 (1989).

[23] C. Travier, Nucl. Instrum. Methods Phys. Res., Sect. A 340, 26 (1994).

[24] Los Alamos Accelerator Code Group, http://laacg.lanl.gov/ laacg/services/services.phtml.

[25] Pulsar Physics, http://www.pulsar.nl/gpt/index.html.

[26] Y. Nishimura, T. Takahashi, K. Sakaue, M. Washio, T. Takatomi, and J. Urakawa, Proceedings of the 4th International Particle Accelerator Conference, IPAC2013, Shanghai, China, 2013 (JACoW, Shanghai, China, 2013), pp. 2753-2755. 